\title{
Significados del número natural en libros de texto mexicanos: un análisis descriptivo
}

\author{
Meanings of the natural number in Mexican textbooks: a \\ descriptive analysis
}

\author{
Lizzet Morales-Garcia ${ }^{1}$ \\ Catalina Navarro Sandoval ${ }^{2}$ \\ Danilo Díaz-Levicoy ${ }^{3}$
}

\begin{abstract}
Resumen: La investigación tiene como objetivo caracterizar el significado pretendido para el tratamiento del número natural, en libros de texto de matemáticas, correspondientes a los tres primeros grados de la Educación Primaria en México. Para el análisis de las tareas, se retoman elementos teóricos y metodológicos del Enfoque Ontosemiótico de la Cognición e Instrucción Matemáticos (EOS), específicamente lo relacionado con la configuración epistémica y los niveles de análisis de la actividad matemática. Los resultados muestran diferencias entre los tres cursos, en cuanto a la presencia de los significados del número natural. En primer grado, se presentan tareas sobre los significados de secuencia numérica, cardinal, ordinal y operacional. En segundo grado, se aborda el significado simbólico, cardinal, secuencia numérica y operacional. En tercer grado, se promueven los significados operacional, cardinal
\end{abstract}

Fecha de recepción: 24 de septiembre de 2019. Fecha de aceptación: 16 de abril de 2021.

1 Facultad de Matemáticas, Universidad Autónoma de Guerrero, México. Imgarcia@uagro.mx, orcid. org/0000-0002-2295-2278

2 Facultad de Matemáticas, Universidad Autónoma de Guerrero, México. catalinans@uagro.mx, orcid. org/0000-0001-5214-0062

3 Facultad de Ciencias Básicas, Universidad Católica del Maule, Chile. dddiaz01@hotmail.com, orcid. org/0000-0001-8371-7899 
y simbólico. Asimismo, se observa la ausencia del estudio explícito de objetos clave, como lo son, unidad, decena, centena, y unidad de millar.

Palabras clave: análisis de tareas, número natural, significado.

\begin{abstract}
The research aims to characterize the pretended meaning for the treatment of natural number, in math textbooks corresponding to the first three grades of Primary Education in Mexico. For task analysis are used theoretical and methodological elements of the Onto-Semiotic Approach and Math Instruction (OSA), specifically related to epistemic configuration and the math activity analysis level. The results show differences between the three courses, in terms of the presence of the natural numbers meanings. In first grade, the tasks presented allude the meaning of numerical sequence, cardinal, ordinal and operational. In second grade, the tasks presented allude the meaning symbolic, cardinal, numerical sequence and operational. Finally, in third grade, the tasks presented allude the meaning operational, cardinal and symbolic meaning. Likewise, the explicit study absence of the key objects, such as, unity, tens, hundreds, and thousands unit, is observed.
\end{abstract}

Keywords: task analysis, natural number, meaning.

\title{
1. INTRODUCCIÓN
}

En Educación Primaria se han realizado diversas investigaciones sobre el análisis de libros de texto de matemáticas, donde se atienden áreas de conocimiento específicas, tales como: Aritmética (Campistrous et al., 2013; Ceballos y Blanco, 2008; Konic et al., 2010; Orrantia et al., 2005; Rodríguez-Nieto et al., 2019), Algebra (Aké y Godino, 2018; Cárcamo et al., 2018), Geometría (Becerra y González, 2016; Godino y Ruiz, 2002; Guillen et al., 2009; Kiener et al., 2013; Ruesga et al., 2006) y Probabilidad y Estadística (Alsina y Vásquez, 2016; Díaz-Levicoy et al., 2015; Díaz-Levicoy et al., 2015; Díaz-Levicoy et al., 2017; Díaz-Levicoy et al., 2020; Giacomone et al., 2018; Salcedo y Ramírez, 2016).

En aritmética, un aspecto que ha sido estudiado es el significado del número, dado que, es considerado como un componente importante para el desarrollo del sentido numérico (Cid et al., 2003; Godino et al., 2009; National Council of 
Teachers of Mathematics-NCTM, 1989; Secretaría de Educación Pública (SEP), 2011a). En ese sentido, existen diversas investigaciones donde el foco de atención es el análisis de tareas referidas al tratamiento del número natural en Educación Primaria (Block y Álvarez ,1999; Cortez, 2015; Morales-Garcia, 2015; Navarro, 2015; Salgado y Salinas, 2009; Soto, 2016), así como la pluralidad de significados asociados al número natural, tomando como referencia los contextos y prácticas de uso (Alcalde et al., 2014; Godino et al., 2009; Rico et al., 2008). Pero poco se ha estudiado sobre la representatividad del significado del número natural en libros de texto de matemáticas en Educación Primaria. Por tal motivo, la pregunta de investigación que interesa responder es: ¿Cuáles son los significados pretendidos del número natural en libros de texto de primaria? Por tal motivo, el objetivo de la investigación es caracterizar el significado pretendido del número natural en los libros de texto de matemática correspondientes a los tres primeros grados de Educación Primaria en México.

La importancia del análisis del libro de texto de matemáticas radica, principalmente, en el uso que los profesores y las mismas reformas educativas le han dado, convirtiéndolos en "mediadores entre el currículo y el aula" (Ceballos y Blanco, 2008, p. 1). Por otra parte, de acuerdo con Braga y Belver (2016), el estudio de estos es prudente, debido a que permite informar sobre la potencialidad de dicho material didáctico, y con ello favorecer la reflexión curricular. Ahora bien, respecto al análisis de libros de texto sobre el concepto número natural en nivel primario, investigadores como Salgado y Salinas (2009) estudiaron algunas actividades referidas al número, en libros de texto de editoriales distintas, como resultado los autores señalan que la metodología empleada por las editoriales se basó en el aprendizaje memorístico y la repetición de ejercicios. Por otra parte, Block y Álvarez (1999), reportan algunos cambios en las propuestas oficiales para la enseñanza del número en primer grado en México entre 1960 y 1990, mencionando que es en los años noventa cuando se aborda el significado de los conceptos, en distintos contextos de uso.

\section{ENFOQUE ONTOSEMIÓTICO DE LA COGNICIÓN E INSTRUCCIÓN MATEMÁTICOS (EOS)}

En el EOS, una de las nociones importantes es la de significado de un objeto matemático, entendido desde un punto de vista pragmático, es decir, depende del contexto donde se utilice, y por tanto del sistema de prácticas matemáticas 
asociadas al mismo. De acuerdo con Godino et al. (2019) es "la correspondencia entre un objeto y el sistema de prácticas donde interviene tal objeto" (p. 7). En ese sentido, un objeto matemático puede tener más de un significado (Godino et al., 2017), esta diversidad de significados se puede observar en el trabajo citado para el caso de la proporcionalidad; así como en Godino et al. (2011), donde analizan algunos significados para el número natural. Desde el EOS, se hace énfasis en dos tipos de significado, el personal y el institucional; el primero está relacionado con el sistema de prácticas matemáticas realizado por una persona, como cuando un estudiante resuelve una tarea matemática, y el segundo está relacionado con el sistema de prácticas matemáticas efectuadas por una institución. De acuerdo con Godino y Batanero (1994), "Ios libros de texto pueden ser considerados como instituciones portadoras de aspectos parciales del significado de los objetos matemáticos correspondientes" (p. 14). Por tal motivo, la noción de significado institucional de un objeto matemático en un libro de texto, conlleva el reconocimiento de una pluralidad de significados, que se hacen evidentes mediante el sistema de prácticas institucionales de donde emerge el objeto matemático. Es decir, el reconocimiento del significado pretendido en libros de texto para el número natural y un método para identificar o delimitar este significado es por medio del análisis de los sistemas de prácticas y las configuraciones ontosemióticas implicadas en las mismas (Godino et al., 2019).

Una herramienta del EOS que permite estudiar los significados y prácticas matemáticas institucionales asociadas a un objeto matemático, es la configuración ontosemiótica. En particular, mediante esta herramienta se puede hacer un análisis epistémico de la actividad matemática puesta en juego en la resolución de problemas (Godino, 2017). En este caso, se utiliza para identificar la trama de objetos primarios (situaciones-problema, lenguajes, conceptos, procedimientos, proposiciones y argumentos) que emergen en la resolución de tareas asociadas al tratamiento del número natural, en un libro de texto. De acuerdo con Godino et al. (2017) y con el objetivo de la investigación, se presenta la siguiente distinción entre los objetos primarios utilizados para el análisis de tareas. Ver figura 1. 


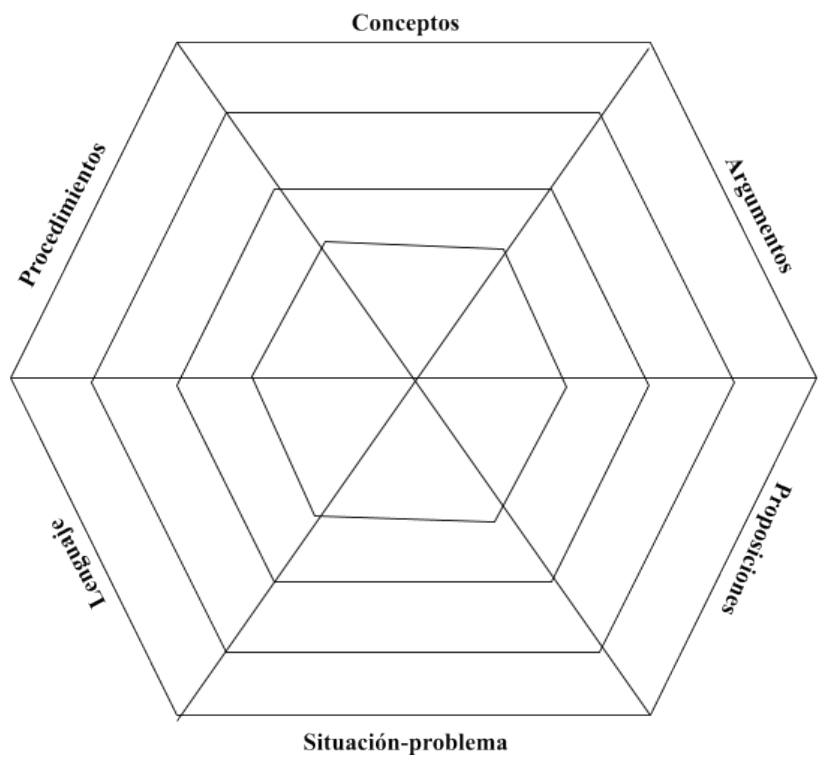

Figura 1. Configuración epistémica adaptado de (Godino et al., 2006, p. 11).

- Situaciones-problema. Son aplicaciones tanto extra-matemáticas como intra-matemáticas del objeto matemático. Para esta investigación se identifican aquellas situaciones-problema que involucren contextos de uso del número natural.

- Lenguajes. Son términos, expresiones, notaciones, gráficos en sus diversos registros (escrito, oral, gestual, etc.). En este caso, se identifican términos, expresiones, notaciones, representaciones gráficas, etcétera; asociadas con el número natural. Por ejemplo, la expresión verbal (uno, dos, tres, ..., diez).

- Conceptos-definición. Introducidos mediante definiciones o descripciones. En este caso, aquellos conceptos-definición que se promuevan en la resolución de la tarea. Por ejemplo, sistema de numeración, valor posicional, número ordinal, cardinalidad, entre otros.

- Procedimientos. Son los algoritmos, operaciones, técnicas de cálculo utilizados en la actividad matemática. Por ejemplo, el uso del conteo de colecciones de objetos, la descomposición aditiva de un número de dos o más cifras, el conteo ordinal, etcétera.

- Argumentos. Son enunciados para validar o explicar las proposiciones y procedimientos usados para resolver una tarea. Por ejemplo, cuando se quiere justificar el procedimiento para realizar la descomposición aditiva de un número de tres cifras. 
- Proposiciones. Son enunciados sobre conceptos empleados en la resolución de la tarea. Por ejemplo, la relación entre unidades, decenas y centenas, la lectura y escritura de números, la comparación de cardinales, etcétera.

Por lo tanto, se efectuó un análisis epistémico debido a que interesa centrarse en la configuración de objetos que se activan en las prácticas matemáticas necesarias para resolver una tarea. En este punto, es importante resaltar el significado institucional del objeto matemático con base en las prácticas matemáticas pretendidas en los libros de texto seleccionados para la investigación.

\section{METODOLOGÍA}

El trabajo de investigación es de tipo cualitativo, de nivel descriptivo y se centra en el análisis de los libros de texto Desafíos matemáticos, que de acuerdo con la SEP (2011a) eran utilizados en el segundo periodo escolar, es decir, $1^{\circ}, 2^{\circ}$ y $3^{\circ}$ de la Educación Primaria en México. En la tabla 1, se presenta información sobre los textos analizados.

Tabla 1. Libros de texto de $1^{\circ}, 2^{\circ}$ y $3^{\circ}$ de la Educación Primaria en México

\begin{tabular}{cl}
\hline Grado escolar & \multicolumn{1}{c}{ Libro de texto } \\
\hline $1^{\circ}$ & Desafíos Matemáticos primer grado. Libro para el alumno (SEP, 2014a) \\
$2^{\circ}$ & Desafíos Matemáticos segundo grado. Libro para el alumno (SEP, 2014b) \\
$3^{\circ}$ & Desafíos Matemáticos tercer grado. Libro para el alumno (SEP, 2014c) \\
\hline
\end{tabular}

De acuerdo con los Programas de Estudio 2011 (SEP, 2011b, 2011c; 2011d) para el eje sentido numérico y pensamiento algebraico se tiene como objetivo que "el alumno lee, escribe y compara números naturales de hasta cuatro cifras" (SEP, 2011b p. 72). El eje está organizado en ocho temas, y para esta investigación se analiza lo referido al tema "números y sistemas de numeración" así como los contenidos y tareas propuestas para el tratamiento del mismo, en los tres grados escolares. En la tabla 2, se muestran los aprendizajes esperados para el tratamiento del número natural en cada grado escolar. 
Tabla 2. Contenidos para el tratamiento de número natural en el segundo periodo escolar de la Educación Primaria en México

\begin{tabular}{|c|c|}
\hline $\begin{array}{l}\text { Grado } \\
\text { escolar }\end{array}$ & Aprendizajes esperados/ contenidos \\
\hline $1^{\circ}$ & $\begin{array}{l}\text { - Comparación de colecciones pequeñas con base en su cardinalidad (SEP, } \\
\text { - Expresión oral de la sucesión numérica ascendente y descendente de } 1 \text { en } 1 \text {, } \\
\text { a partir de un número dado (SEP, 2011b, p. 84). } \\
\text { - Escritura de la sucesión numérica hasta el } 30 \text { (SEP, 2011b, p. 84). } \\
\text { - Identificación y uso de los números ordinales para colocar objetos o para } \\
\text { indicar el lugar que ocupan dentro de una colección de hasta } 10 \text { elementos } \\
\text { (SEP, 2011b, p. 84). } \\
\text { - Identificación de regularidades de la sucesión numérica del } 0 \text { al } 100 \text { al } \\
\text { - } \text { organizarla en intervalos de } 10 \text { (SEP, 2011b, p. 85). } \\
\text { - de números de hasta dos cifras (SEP, 2011b, p. 85). } \\
\text { - Descomposición de números de dos cifras como sumas de un sumando que } \\
\text { se repite y algo más (SEP, 2011b, p. 86). }\end{array}$ \\
\hline $2^{\circ}$ & $\begin{array}{l}\text { - Identificación de las características de hasta tres cifras que forman un } \\
\text { número para compararlo con otros números (SEP, 2011c, p. 84). } \\
\text { - } \text { Elaboración de estrategias para facilitar el conteo de una colección numerosa } \\
\text { (SEP, 2011c, p. 84). } \\
\text { - } \text { Determinación del valor de las cifras en función de su posición en la } \\
\text { - escritura de un número (SEP, 2011c, p. 85). } \\
\text { - } \text { Orden y comparación de números hasta de tres cifras (SEP, 2011c, p. 85). } \\
\text { - } \text { númerificación de algunas diferencias entre la numeración oral y la escrita con } \\
\text { - } \text { decritura de números mediante descomposiciones aditivas en centenas, } \\
\text { - Producción de sucesiones orales y escritas, ascendentes y descendentes, de } \\
100 \text { en } 100 \text {. Anticipaciones a partir de las regularidades (SEP, 2011c, p. 86). }\end{array}$ \\
\hline $3^{\circ}$ & $\begin{array}{l}\text { - Uso de la descomposición de números en unidades, decenas, centenas y } \\
\text { unidades de millar para resolver diversos problemas (SEP, 2011d, p. 74). } \\
\text { - Relación de la escritura de los números con las cifras y su nombre, a través } \\
\text { de su descomposición aditiva (SEP, 2011d, p. 74). }\end{array}$ \\
\hline
\end{tabular}

En total se identificaron 77 tareas o consignas (parte del libro de texto donde se muestra la situación-problema a resolver) sobre números naturales. Para el análisis se tomó en cuenta los sistemas de prácticas puestos en juego en la resolución de las tareas, y la exploración sistemática de los contextos de uso del 
objeto matemático (Godino et al., 2019), en este caso el número natural. Una herramienta que posibilita el análisis de los sistemas de prácticas asociados a la resolución de una situación-problema, son los dos niveles de análisis de la actividad matemática utilizados en Godino et al. (2017), estos niveles de análisis permiten relacionar las nociones de significado institucional, sistema de prácticas y configuración de objetos.

El primer nivel consiste en el análisis fenomenológico-antropológico el cual permite identificar fenómenos o situaciones-problema, asociadas con el uso de número natural, así como el análisis de las prácticas matemáticas que emergen en la resolución de tareas. De acuerdo con Godino et al. (2017) la finalidad de este análisis es caracterizar la diversidad de significados parciales de un objeto matemático y su articulación en un sistema global que sirva de referencia en el diseño y gestión de los procesos de estudio.

Por otra parte, el segundo nivel de análisis el análisis ontosemiótico se centra en identificar la trama de objetos matemáticos y relaciones que se ponen en juego en cada una de las prácticas matemáticas; que constituyen el sistema de prácticas esperadas en la resolución de la situación-problema. Para la identificación de esa trama de objetos, se utilizó la herramienta configuración ontosemiótica presentada en Godino et al. (2017), en la cual se identifican objetos primarios emergentes en la resolución de tareas, donde se resaltan los siguientes elementos:

- Enunciado y secuencia de prácticas elementales para resolver la tarea. En la cual se organiza el enunciado y secuencia de prácticas (operativas y discursivas) que resultan importantes en la resolución de la tarea.

- Uso e intencionalidad de las prácticas. Cuyo propósito es reconocer la función de cada práctica matemática en la resolución de la tarea planteada.

- Objetos referidos en las prácticas. Se muestra la trama de objetos primarios (conceptos, lenguajes, procedimientos, proposiciones y argumentos) identificados en cada una de las prácticas matemáticas.

La finalidad de este segundo nivel de análisis de acuerdo con Godino et al. (2017) es desvelar la complejidad ontosemiótica de un objeto, como factor explicativo de los conflictos y dificultades de su aprendizaje. Lo anterior servirá de base para la estructuración de la configuración epistémica de los objetos primarios involucrados en la actividad matemática. 


\section{SIGNIFICADOS DE LOS NÚMEROS NATURALES}

Tomando como referencia a Alcalde et al. (2014), Cid et al. (2003) y Rico et al. (2008) se pueden señalar seis contextos numéricos asociados al uso de los números naturales.

- Secuencia numérica. Consiste en el uso del número para indicar el orden para recitar o escribir la secuencia de números naturales. Por ejemplo, tareas donde se pide completar o recitar la secuencia numérica del uno al cien.

- Cardinal. Es asociado con el uso del número para indicar cuántos elementos hay en un conjunto. Por ejemplo, cuando se pide determinar cuántos estudiantes hay en un salón de clases.

- Ordinal. Los números se utilizan para indicar la posición de un objeto en un conjunto ordenado. Por ejemplo, cuando se pide indicar la posición en la que llega un competidor en una carrera.

- Simbólico. En este caso, los números se utilizan a modo de etiqueta o código para identificar o codificar objetos. Por ejemplo, los números en la camiseta de los jugadores o bien los números en una tarjeta bancaria.

- Operacional. Se resalta el uso de los números para realizar operaciones aritméticas. Por ejemplo, aquellas tareas donde se le pide a un estudiante determinar el resultado de $7+3$.

- Medida. Entre otros aspectos, el uso de los números para indicar cuánto mide un objeto o persona. Como cuando se pide determinar la estatura de un estudiante.

La resolución de las tareas planteadas para cada significado, muestra una secuencia de prácticas propias de cada contexto de uso. Todo lo anterior en conjunto, corresponde al análisis fenomenológico-antropológico (nivel 1). Mientras que, el análisis ontosemiótico corresponde a un análisis más fino de cada una de las prácticas (nivel 2) y para ejemplificarlo se emplea la configuración ontosemiótica (tabla 3). A continuación, se muestra el análisis de algunas tareas.

\subsection{SigNIFICADO DEL NÚMERO NATURAL COMO SECUENCIA NUMÉRICA}

En la figura 2, se muestra una tarea donde se hace uso de una canción infantil, para introducir las palabras numéricas del uno al diez y el orden para recitar las mismas. 


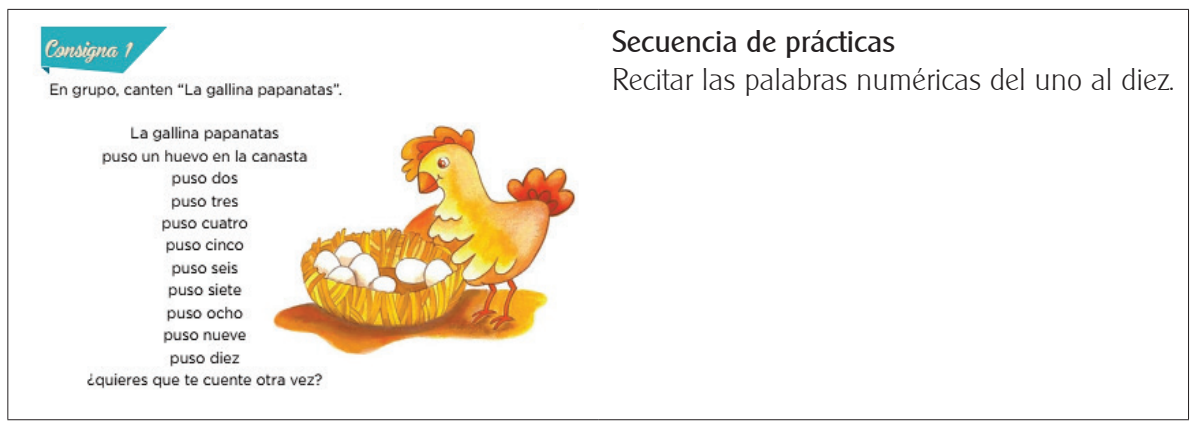

Figura 2. Tarea en el contexto de secuencia numérica (SEP, 2014a, p. 13).

En el lado izquierdo se muestra la tarea analizada, mientras que del lado derecho se presenta la secuencia de prácticas (operativas y discursivas) esperadas para la ejecución de la misma. La tarea presenta las palabras numéricas del uno al diez, y el orden en que se recitan las mismas. La secuencia de prácticas está enfocada en el recitado de las palabras numéricas del uno al diez. Por tal motivo, en el análisis ontosemiótico se analizan prácticas cuyos objetos primarios intervinientes son: la secuencia numérica (concepto), la expresión verbal (lenguaje), y el recitado de la secuencia numérica (procedimiento). El análisis de las prácticas se puede ver en la tabla 3.

Tabla 3. Configuración ontosemiótica de la tarea

\begin{tabular}{lll}
\hline $\begin{array}{l}\text { Enunciado y secuencia } \\
\text { de prácticas elementales } \\
\text { para resolver la tarea }\end{array}$ & $\begin{array}{l}\text { Uso e intencionalidad de } \\
\text { las prácticas }\end{array}$ & Objetos referidos en las prácticas \\
\hline $\begin{array}{l}\text { Recitar las palabras } \\
\text { numéricas del uno al }\end{array}$ & $\begin{array}{l}\text { Reconocer las palabras } \\
\text { numéricas y la secuencia de }\end{array}$ & $\begin{array}{l}\text { Conceptos: secuencia numérica (primer } \\
\text { elemento, segundo, etcétera). } \\
\text { diez. }\end{array}$ \\
& las mismas, para los & $\begin{array}{l}\text { Lenguaje: expresión verbal de los números. } \\
\text { numecedimientos: recitado de la secuencia } \\
\text { de palabras numéricas. }\end{array}$ \\
\hline
\end{tabular}

\subsection{SIGNIFICADO DEL NÚMERO NATURAL COMO CARDINAL}

En la figura 3, se muestra el análisis de una tarea en el contexto cardinal, en la que se pide contestar tres preguntas, sobre la cantidad de objetos en un salón de clases. En este caso, antes de presentar la secuencia de prácticas, se muestra 
la respuesta esperada a las preguntas planteadas en la tarea. Mientras que, en la secuencia de prácticas se muestran los pasos que el estudiante seguiría para llegar a la respuesta esperada.

\begin{tabular}{|c|c|}
\hline $\begin{array}{l}\text { En grupo, contesten las sigulentes preguntas con base en } \\
\text { la Imagen. } \\
\text { - En este salón, ela cantidad de niraas es Igual a la de } \\
\text { - nirosos? } \\
\text { alu cantidad de pupitres es Igual a la cantidad de } \\
\text { - LLa cantidad de llbros es Igual a la cantidad de } \\
\text { alumnos? }\end{array}$ & $\begin{array}{l}\text { Respuesta esperada } \\
\text { En este salón, cla cantidad de niñas es igual a la de ni- } \\
\text { ños? No } \\
\text { ¿La cantidad de pupitres es igual a la cantidad de alum- } \\
\text { nos? No } \\
\text { ¿La cantidad de libros es igual a la cantidad de alumnos? } \\
\text { Sí } \\
\text { Secuencia de prácticas para la resolución del primer } \\
\text { punto } \\
\text { 1. Observar la imagen mostrada e identificar que hay } \\
\quad \text { cuatro niñas y tres niños (suponemos que los niños no } \\
\text { llevan coletas y las niñas sí). } \\
\text { 2. Debido a que tres no es igual que cuatro. } \\
\text { 3. La cantidad de niñas no es igual a la de niños. }\end{array}$ \\
\hline
\end{tabular}

Figura 3. Tarea en contexto cardinal (SEP, 2014a, p. 10).

El análisis ontosemiótico de las prácticas se puede ver en la configuración ontosemiótica presentada a continuación (tabla 4). Mostrando los distintos objetos primarios que intervienen en la solución de la tarea.

Tabla 4. Configuración ontosemiótica de la tarea

\section{Enunciado y secuencia de prácticas elementales para Uso e intencionalidad de las Objetos referidos en las prácticas resolver la tarea prácticas}

En el salón hay tres niños y cuatro niñas.

Debido a que tres no es igual que cuatro.

La cantidad de niñas no es igual a la de niños.

Distinguir entre niños y niñas. Conceptos: conjunto (de niños y niñas), Contar la cantidad de niños y cardinalidad (número de elementos de niñas en el salón. una colección).

Procedimientos: conteo de uno en uno.

Reconocer que el tres es una cantidad menor que cuatro.

Responder a la tarea
Concepto: número

Proposición: $3 \neq 4$

Argumento: ordenación de los números naturales

Proposición: respuesta a la tarea (3 diferente de 4)

Argumentos: la secuencia de pasos 1 a 3. 


\subsection{SIGNIFICADO ORDINAL DEL NÚMERO NATURAL}

En la figura 4, se presenta el análisis de una tarea en contexto ordinal, donde se pretende identificar la posición que le corresponde a cada coche, tomando como referencia su distancia a la meta. En ella intervienen distintos objetos primarios, como las palabras numéricas ordinales, la secuencia en la que se recitan las palabras numéricas, entre otras.

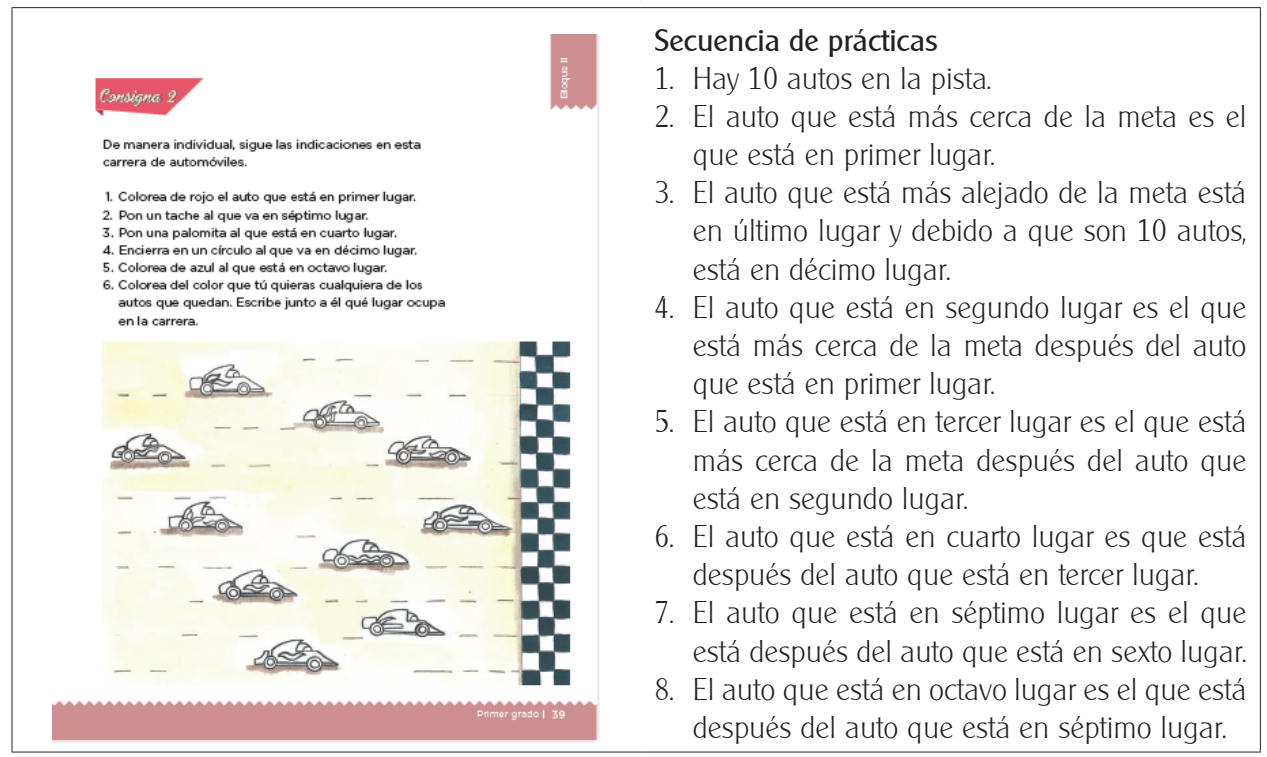

Figura 4. Tarea en contexto ordinal (SEP, 2014a, p. 39).

El análisis ontosemiótico de las prácticas operativas y discursivas utilizadas en la resolución de la tarea, se puede ver en la tabla 5. 
Tabla 5. Configuración ontosemiótica de la tarea

\begin{tabular}{|c|c|c|}
\hline $\begin{array}{l}\text { Enunciado y secuencia de } \\
\text { prácticas elementales para } \\
\text { resolver la tarea }\end{array}$ & $\begin{array}{l}\text { Uso e intencionalidad de } \\
\text { las prácticas }\end{array}$ & Objetos referidos en las prácticas \\
\hline Hay 10 autos en la pista. & $\begin{array}{l}\text { Contar el número de au- } \\
\text { tos en la pista. }\end{array}$ & $\begin{array}{l}\text { Concepto: cardinalidad } \\
\text { Procedimiento: conteo de uno en uno. }\end{array}$ \\
\hline $\begin{array}{l}\text { El auto que está más cerca } \\
\text { de la meta es que el que está } \\
\text { en primer lugar. }\end{array}$ & $\begin{array}{l}\text { Encontrar el auto que está } \\
\text { en primera posición. }\end{array}$ & $\begin{array}{l}\text { Concepto: número ordinal, secuencia de núme- } \\
\text { ros ordinales, distancia; correspondencia entre } \\
\text { distancia y números ordinales. } \\
\text { Proposición: la distancia a la meta determina } \\
\text { la posición del auto. } \\
\text { Lenguajes: palabras numéricas ordinales } \\
\text { Procedimiento: conteo ordinal de una colección. } \\
\text { Argumentos: la secuencia ordinal se corres- } \\
\text { ponde con la ordenación de las distancias de } \\
\text { menor a mayor. }\end{array}$ \\
\hline $\begin{array}{l}\text { El auto que está más alejado } \\
\text { de la meta está en último lu- } \\
\text { gar y debido a que son } 10 \text { ese } \\
\text { auto está en décimo lugar. }\end{array}$ & Reconocer el último lugar. & Concepto: número ordinal, medida y distancia. \\
\hline $\begin{array}{l}\text { El auto que está en segundo } \\
\text { lugar es el que más cerca de } \\
\text { la meta después del auto que } \\
\text { está en primer lugar. }\end{array}$ & $\begin{array}{l}\text { Determinar el segundo } \\
\text { lugar. }\end{array}$ & Concepto: secuencia numérica \\
\hline
\end{tabular}

\subsection{SIGNIFICADO SIMBÓLICO DEL NÚMERO NATURAL}

En la figura 5, se muestra el uso del número natural en un contexto simbólico, particularmente utilizado en una lotería. Asimismo, se analiza la secuencia de prácticas que se ponen en juego para resolver la tarea. 


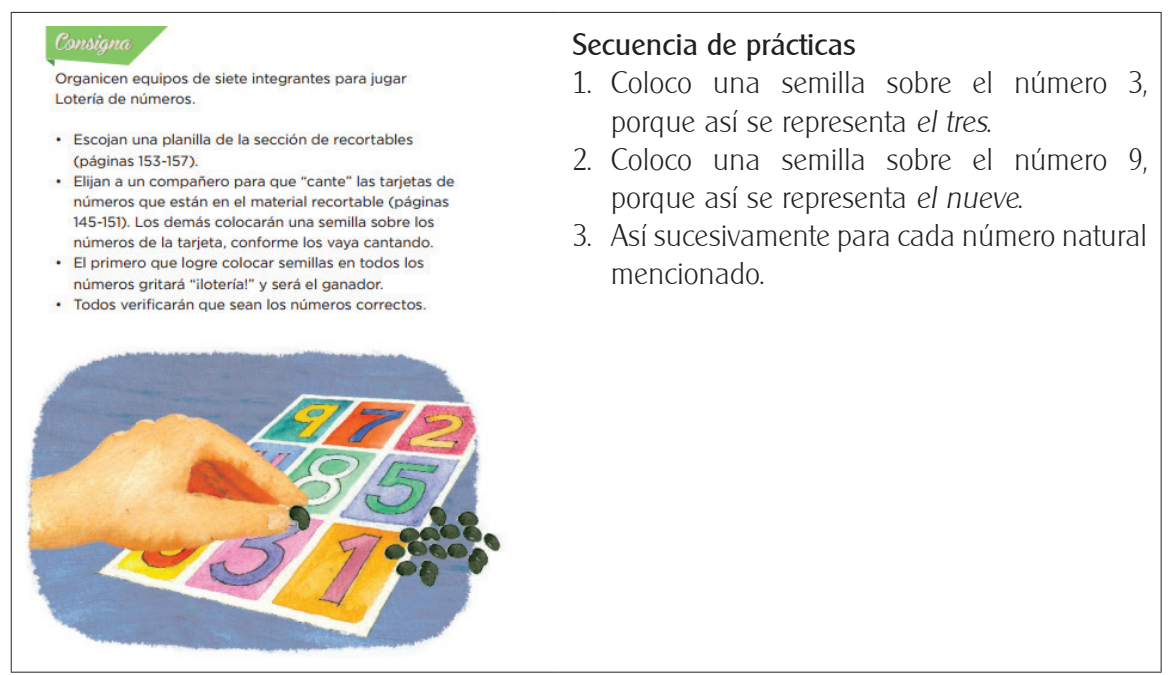

Figura 5. Tarea en el contexto simbólico (SEP, 2014b, p. 76).

A continuación, se presenta la configuración ontosemiótica asociada al análisis ontosemiótico de la tarea (tabla 6).

Tabla 6. Configuración ontosemiótica de la tarea

\begin{tabular}{|c|c|c|}
\hline $\begin{array}{l}\text { Enunciado y secuencia de } \\
\text { prácticas elementales para } \\
\text { resolver la tarea }\end{array}$ & $\begin{array}{l}\text { Uso e intencionalidad de las } \\
\text { prácticas }\end{array}$ & $\begin{array}{l}\text { Objetos referidos en las } \\
\text { prácticas }\end{array}$ \\
\hline $\begin{array}{l}\text { Coloco una semilla sobre el } \\
\text { número } 3 \text {, porque así se re- } \\
\text { presenta "tres". }\end{array}$ & $\begin{array}{l}\text { Identificar la escritura correcta } \\
\text { del "tres". }\end{array}$ & $\begin{array}{l}\text { Lenguaje: expresión verbal y es- } \\
\text { crita de números de una cifra. }\end{array}$ \\
\hline $\begin{array}{l}\text { Coloco una semilla sobre el } \\
9 \text {, porque así se representa } \\
\text { "nueve". }\end{array}$ & $\begin{array}{l}\text { Identificar la escritura correcta } \\
\text { del "nueve". }\end{array}$ & $\begin{array}{l}\text { Procedimiento: relacionar la ex- } \\
\text { presión verbal con la expresión } \\
\text { escrita. }\end{array}$ \\
\hline $\begin{array}{l}\text { Así sucesivamente para cada } \\
\text { número natural mencionado. }\end{array}$ & $\begin{array}{l}\text { Identificar la escritura correcta } \\
\text { de números de una cifras. }\end{array}$ & $\begin{array}{l}\text { Lenguaje: expresión verbal y es- } \\
\text { crita de números de una cifra. } \\
\text { Concepto: unidad }\end{array}$ \\
\hline
\end{tabular}




\subsection{SIGNIFICADO OPERACIONAL DEL NÚMERO NATURAL}

En la figura 6, se presenta una tarea del número natural en contexto operacional. En la que se utilizan números de dos y una cifra para descomponer otros números como la suma de decenas y unidades.

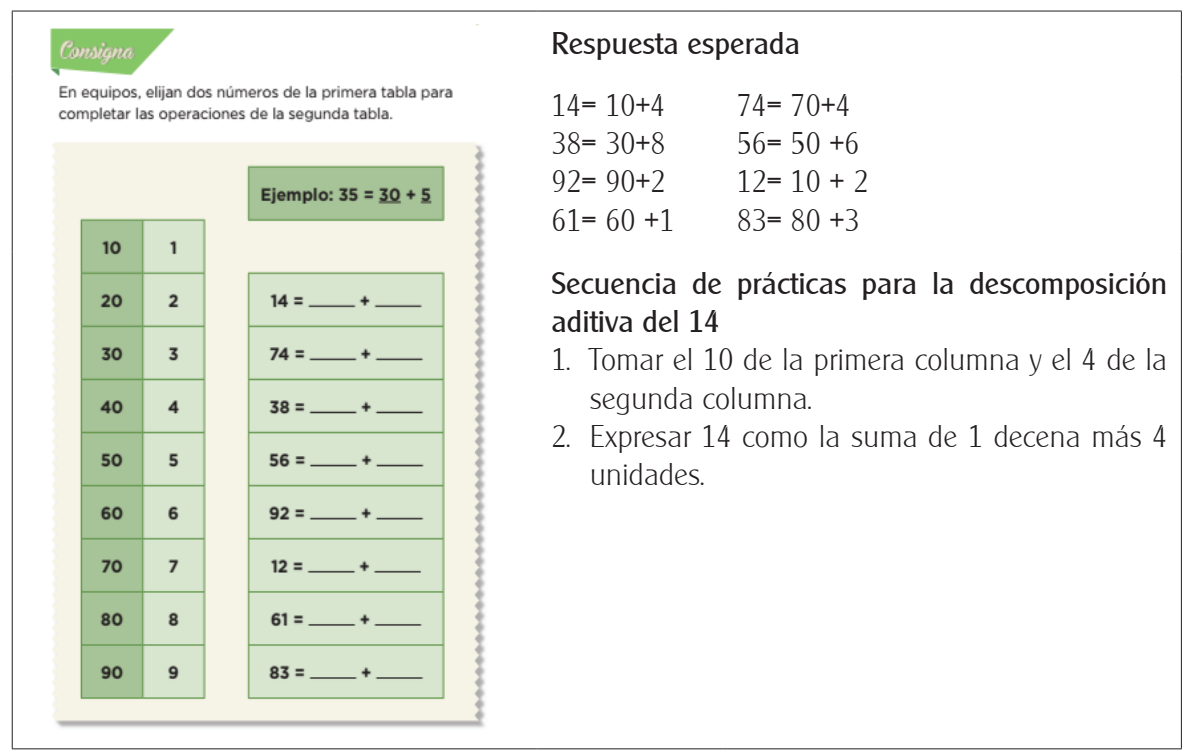

Figura 6. Tarea en el contexto operacional (SEP, 2014a, p. 84).

El análisis ontosemiótico de las prácticas se presenta a continuación.

Tabla 7. Configuración ontosemiótica de la tarea

\begin{tabular}{|c|c|c|}
\hline $\begin{array}{l}\text { Enunciado y secuencia de } \\
\text { prácticas elementales para } \\
\text { resolver la tarea }\end{array}$ & $\begin{array}{l}\text { Uso e intencionalidad de las } \\
\text { prácticas }\end{array}$ & Objetos referidos en las prácticas \\
\hline $\begin{array}{l}\text { Tomar el } 10 \text { de la primera colum- } \\
\text { na y el } 4 \text { de la segunda columna. }\end{array}$ & $\begin{array}{l}\text { Reconocer los números para } \\
\text { la expresión aditiva del } 14 .\end{array}$ & Concepto: descomposición aditiva \\
\hline $\begin{array}{l}\text { Expresar } 14 \text { como la suma de } 1 \\
\text { decena más } 4 \text { unidades. }\end{array}$ & $\begin{array}{l}\text { Identificar la expresión aditiva } \\
\text { del } 14 .\end{array}$ & $\begin{array}{l}\text { Concepto: suma, decena, y unidad. } \\
\text { Procedimiento: expresar en términos } \\
\text { de decenas y unidades el } 14 \text {. }\end{array}$ \\
\hline
\end{tabular}




\section{RESULTADOS}

A continuación, se muestran resultados generales de la cantidad de tareas propuestas para el tratamiento del número natural en $1^{\circ}, 2^{\circ}$ y $3^{\circ}$ de primaria. Ver tabla 8.

Tabla 8. Número de tareas propuestas para cada significado

\begin{tabular}{ccccccc}
\hline \multirow{2}{*}{ Grado } & \multicolumn{6}{c}{ Significado } \\
\cline { 2 - 7 } & $\begin{array}{c}\text { Secuencia } \\
\text { Numérica }\end{array}$ & Cardinal & Ordinal & Simbólico & Operacional & Medida \\
\hline $1^{\circ}$ & 17 & 19 & 3 & 0 & 1 & 0 \\
$2^{\circ}$ & 5 & 17 & 0 & 3 & 5 & 0 \\
$3^{\circ}$ & 0 & 2 & 0 & 2 & 3 & 0 \\
\hline Total & 22 & 38 & 3 & 5 & 9 & 0 \\
\hline
\end{tabular}

En la tabla se evidencia que, de las 77 tareas propuestas para el tratamiento del número natural, 22 enfatizan en el significado del número como secuencia numérica, 38 en el significado cardinal, 3 en el significado ordinal, 5 en el significado simbólico, 9 en el significado operacional y 0 al significado de medida. Esto último, de ninguna manera quiere decir que el significado del número natural como medida no sea abordado en los libros de texto. La justificación es que de los ocho temas que conforman el eje sentido numérico y pensamiento algebraico para esta investigación solo se analizan las tareas del tema números y sistemas de numeración. Mientras que, las tareas referidas al significado del número como medida están incluidas en el eje forma, espacio y medida.

\subsection{CONFIGURACIONES EPISTÉMICAS ASOCIADAS AL NÚMERO NATURAL EN PRIMER GRADO}

De acuerdo con el análisis efectuado para el libro de texto correspondiente al primer grado de Educación Primaria en México (SEP, 2014a), se identificaron tareas referidas al significado del número natural como: secuencia numérica, cardinal, ordinal y operacional. En cada uno de los significados y de acuerdo con la secuencia de prácticas y las configuraciones ontosemióticas, se identifican objetos primarios propios de cada significado, organizados en configuraciones epistémicas (figura 7). 


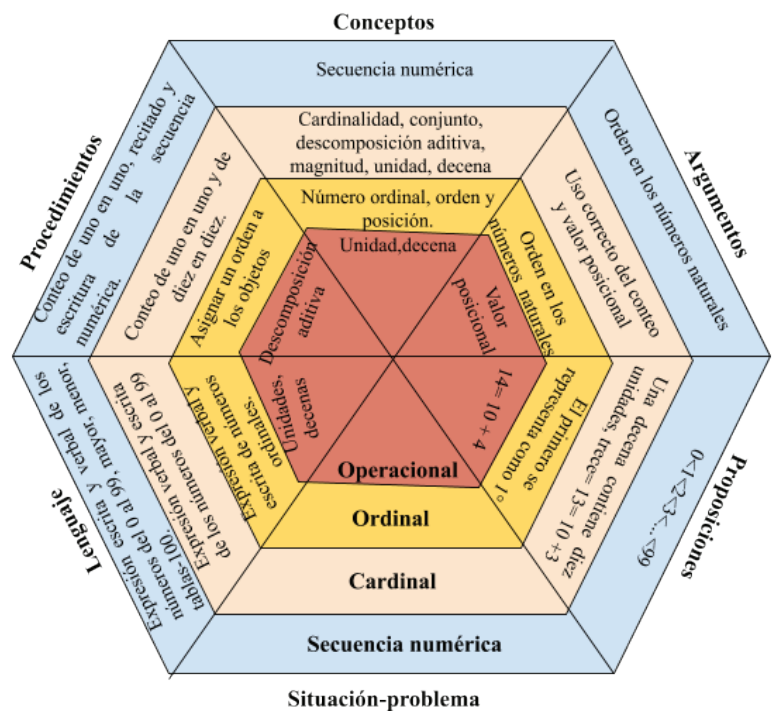

Figura 7. Configuraciones epistémicas del número natural en primer grado de primaria.

En la configuración epistémica del número natural como secuencia numérica, en el objeto lenguaje se identificó el trabajo con la expresión verbal y escrita de números del 0 al 99, como apoyo para el trabajo con dicho aspecto en algunas tareas se proponen el uso de las tabla-100. Por otra parte, en el caso de los procedimientos se hace énfasis en el conteo y la escritura ordenada de los números del 0 al 99, proponiendo para ello, actividades de ejercitación de la secuencia numérica y en algunos casos el uso de material concreto. Se aborda el concepto de secuencia numérica. En la parte de argumentos el análisis arrojó que las secuencias de prácticas se basan en el orden de la secuencia numérica, en ese sentido las proposiciones se relacionan con el orden en que se escribe la misma, es decir, $0<1<2<3 \ldots<99$, así como la expresión verbal de los números (uno, dos, tres, ..., noventa y nueve).

Por otra parte, en la configuración epistémica del número natural como cardinal, se identificaron tareas relacionadas con el conteo de colecciones de objetos (frijolitos, dinero, naranjas, etc.), así como tareas que abordan el valor posicional de números de dos cifras. En ese sentido, en el apartado de lenguaje se tratan aspectos como la expresión verbal y escrita de números del 0 al 99, así como el uso de fichas de colores para el trabajo con el valor posicional. Mientras que, el procedimiento que predomina en la resolución de las tareas, es el conteo 
de uno en uno y de diez en diez. Respecto a los conceptos se nota la emergencia del concepto cardinalidad, conjunto, descomposición aditiva, magnitud, unidad, decena. Ahora, para el caso de los argumentos existen dos predominantes, aquellos relacionados con el valor posicional de números de dos cifras, y realizar correctamente el conteo de una colección de objetos. Finalmente, en el caso de las proposiciones, estas se relacionan con la conversión entre la expresión verbal y la escrita, por ejemplo, trece se representa como 13, así como el valor posicional, es decir, una decena está conformada por diez unidades.

Para la configuración epistémica del número natural respecto del significado ordinal, el libro de texto presenta tareas donde se aborda el uso y la ejercitación de números ordinales. En ese sentido, en el apartado de lenguaje se trabajan las expresiones verbales (primero, segundo, tercero, cuarto, quinto, sexto, séptimo, octavo, noveno y décimo) y las expresiones numéricas $\left(1^{\circ}, 2^{\circ}, 3^{\circ}, 4^{\circ}, 5^{\circ}, 6^{\circ}, 7^{\circ}\right.$, $8^{\circ}, 9^{\circ}$ y $10^{\circ}$ ) de números ordinales. El procedimiento utilizado gira en torno a ordenar los objetos para su posterior conteo en forma ordinal. Los conceptos identificados en las tareas son número ordinal, orden, y posición. Los argumentos recaen principalmente en el orden en los números naturales como factor importante para la asignación del correspondiente número ordinal. En el caso de las proposiciones identificadas, estas guardan relación entre expresiones verbales y numéricas de números ordinales. Por ejemplo, primero se representa como $1^{\circ}$ y así sucesivamente para cada número ordinal trabajado.

Finalmente, en la configuración epistémica del número natural en su significado operacional, el libro de texto presenta una tarea donde se tiene como lenguaje la descomposición aditiva de números naturales de dos cifras en términos de unidades y decenas. Por otra parte, se presenta como procedimiento la descomposición aditiva, como argumento el valor posicional de las cifras de un número. Y finalmente como proposiciones la descomposición aditiva del 14 como $10+4$.

\subsection{CONFIGURACIONES EPISTÉMICAS ASOCIADAS AL NÚMERO NATURAL EN SEGUNDO GRADO}

En cuanto a la organización del tema números naturales, en el libro de texto de segundo grado de Educación Primaria (SEP, 2014b) se plantea la enseñanza de los números naturales del 100 al 999. Presentando trabajo con cuatro significados del número natural. A continuación, en la figura 8 , se señalan las configuraciones epistémicas para el significado simbólico, cardinal, secuencia numérica y operacional del número natural. En cada configuración epistémica 
se destacan las situaciones-problemas, lenguaje, procedimientos, conceptos, argumentos y proposiciones que emergen al trabajar en cada uno.

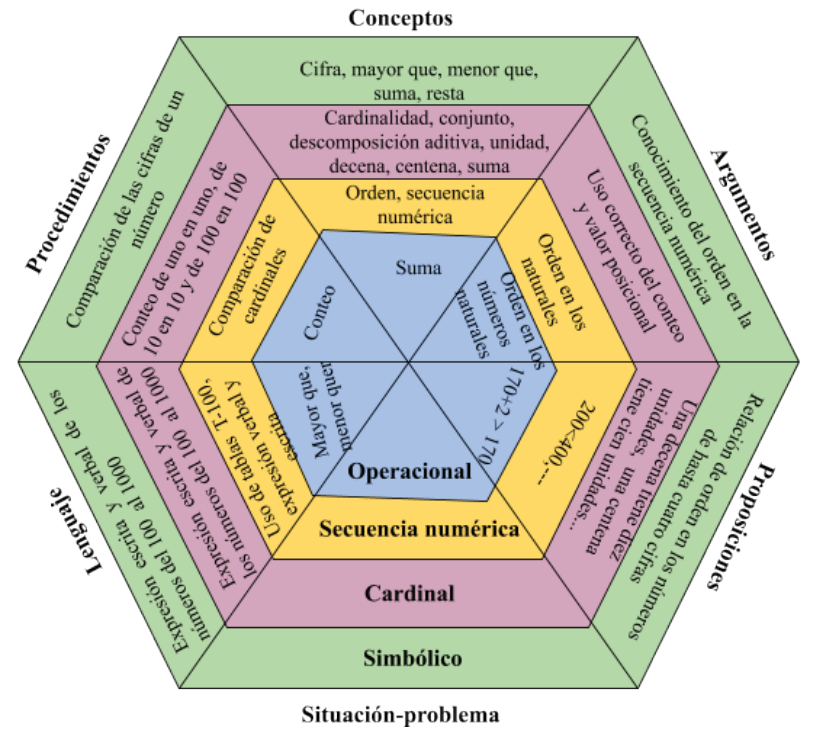

Figura 8. Configuraciones epistémicas del número natural en segundo grado.

En la configuración epistémica del número natural como símbolo, en el objeto lenguaje se muestra el uso de la expresión verbal y escrita de los números naturales hasta el 999, utilizando tarjetas. Para el caso de los procedimientos estos se relacionan con la comparación de las cifras de un número. Los conceptos abordados son cifra, mayor, menor, suma y resta, mientras que los argumentos en que se basa la realización de las tareas están aunados al orden de los números naturales. Finalmente, las proposiciones están relacionadas con determinar si un número se ubica antes o después en la secuencia numérica dada.

Por otra parte, en la configuración epistémica del número natural como cardinal el libro de texto presenta tareas relacionadas con el conteo, la lectura y escritura de números hasta el 999, y la comparación de cardinales. En ese sentido como resultados del análisis se muestra que, en el caso del lenguaje, se trabaja con la expresión verbal y escrita de los números naturales hasta el 999. Por otra parte, los conceptos trabajados son cardinalidad, conjunto, descomposición aditiva, unidad, decena y centena. Los argumentos que se hacen evidentes en la realización de la tarea están asociados a hacer correctamente el 
conteo de una colección de objetos y el valor posicional, y finalmente como proposiciones se tiene que una decena está conformada por diez unidades, una centena conformada por diez decenas o cien unidades.

En el caso de la configuración epistémica del número natural como secuencia numérica se muestra que el libro propone tareas para el tratamiento de la secuencia numérica hasta el 999. En ese sentido, el análisis de las tareas permite conocer que en el apartado lenguaje se promueve el trabajo con la expresión verbal y escrita de los números naturales hasta el 999, así como el uso de las tablas-100. En el caso del procedimiento se logra identificar el trabajo con la comparación de cardinales, y el reconocimiento de características de tramos de las secuencias numéricas para lograr la escritura correcta de la misma. Los conceptos abordados son orden y secuencia numérica. Para el caso de los argumentos, el análisis muestra que se hace referencia al orden en los números naturales, y en el caso de las proposiciones se tienen las siguientes $10<20<30 \ldots<990,400<200$ etcétera.

Finalmente, en la configuración epistémica del significado operacional del número natural, el libro de texto presenta tareas donde se hace uso del número para realizar operaciones básicas de suma y resta. El lenguaje utilizado resalta el uso de la expresión escrita de los números del 0 al 999, el uso de los términos mayor que, menor que. En el caso del procedimiento está el conteo. Como conceptos la suma y en el caso de los argumentos emergentes en la resolución de la tarea, se destacan la aplicación correcta de la suma o resta en la resolución de las tareas, y finalmente las proposiciones están asociadas en indicar que número es menor o mayor, por ejemplo: 170+2 > 170 .

\subsection{CONFIGURACIONES EPISTÉMICAS ASOCIADAS AL NÚMERO NATURAL EN TERCER GRADO}

De acuerdo con el análisis de las tareas propuestas en tercer grado de la Educación Primaria (SEP, 2014c), se identifica que en este grado escolar abordan el significado operacional, cardinal y el simbólico del número natural. La caracterización de los mismos viene dada por el tipo de objetos primarios (situaciones-problema, lenguaje, procedimientos, conceptos, argumentos y proposiciones) que emergen en cada uno de ellos, como ya se mencionó anteriormente esta caracterización se hace evidente mediante la correspondiente configuración epistémica. En la figura 9 se presenta las configuraciones epistémicas para el significado operacional, cardinal y el significado simbólico del número natural identificadas en el libro. 


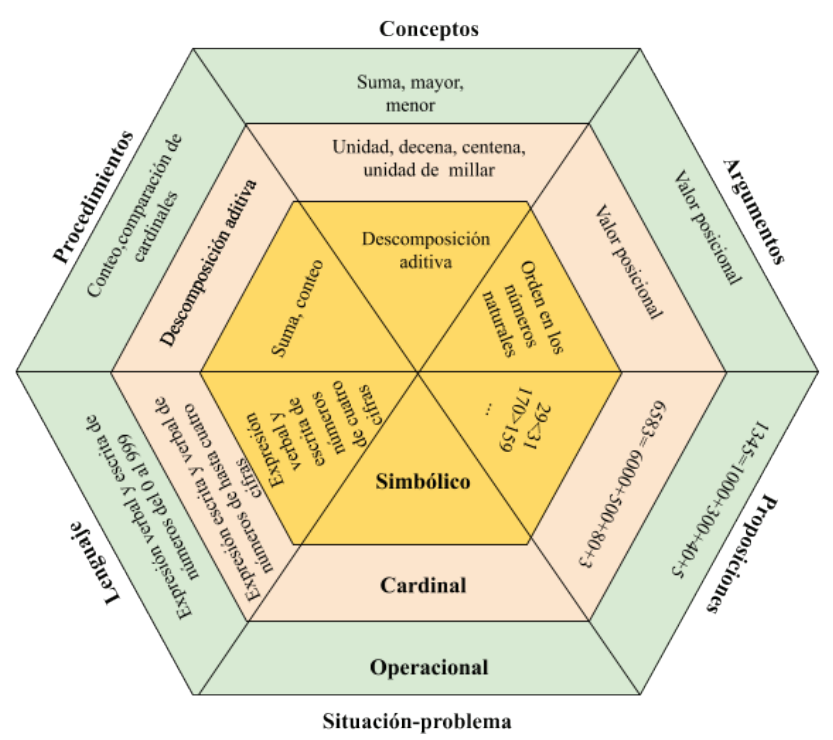

Figura 9. Configuraciones epistémicas del número natural en tercer grado.

Para la configuración epistémica del significado operacional. Las tareas propuestas muestran en el lenguaje el uso de la expresión escrita y verbal de los números del 0 al 999, como procedimiento el conteo y la comparación de cardinales, como conceptos la suma, como argumentos el valor posicional, y como proposiciones la descomposición aditiva de algunos números.

Por otra parte, la configuración epistémica del significado cardinal del número natural, las tareas propuestas para el tratamiento de este significado, están enfocadas específicamente en el valor posicional para números de hasta cuatro cifras. En ese sentido, en el lenguaje se trabaja con la expresión verbal y escrita de números de hasta cuatro cifras, como procedimiento para lo anterior está la descomposición aditiva, mientras que los conceptos son unidades de millar, centenas, decenas y unidades, aunado a lo anterior como argumentos se tiene el valor posicional, y las proposiciones están relacionadas a la descomposición aditiva de algunos números de cuatro cifras, por ejemplo, 6583=6000+500+80+3.

Finalmente, para la configuración epistémica del significado simbólico del número natural, las tareas propuestas se inclinan con la comparación de números naturales, por tal motivo en el apartado de lenguaje se trabaja con la expresión verbal y escrita de números de hasta cuatro cifras, como procedimientos la comparación de cardinales, suma y el conteo, como conceptos el significado de 
mayor, menor y como argumentos aquellos relacionados con el orden de la secuencia numérica y en concordancia con lo anterior como proposiciones se tienen aquellas relacionadas con indicar cuando un número es mayor o menor que otro, por ejemplo, 29<31, 170> 159.

\section{REFLEXIONES}

\subsection{SOBRE LAS CONFIGURACIONES EPISTÉMICAS}

El trabajo estuvo enfocado en caracterizar el significado pretendido del número natural en libros de texto de matemáticas, correspondientes a los tres primeros grados de Educación Primaria en México. El uso de los niveles de análisis de la actividad matemática (Godino et al., 2017) permitió caracterizar la propuesta de enseñanza desde las situaciones-problema, el lenguaje, procedimientos, conceptos, proposiciones y argumentos identificados con el análisis presentado para cada grado escolar.

Con respecto a las situaciones-problema se identificaron 77 tareas que aluden al tratamiento de los distintos significados asociados al número natural, en los tres grados escolares. De las cuales 38 tareas son referidas al significado cardinal, 22 al significado de secuencia numérica, 9 al significado operacional, 5 al significado simbólico y 3 al significado ordinal. El mayor trabajo se concentra en primer y segundo grado con 70 tareas, mientras que en tercer grado solo se proponen 7 .

Con respecto al lenguaje, se propone el trabajo con la expresión verbal y/o escrita de números naturales en primer grado hasta el 100, en segundo grado hasta el 1000 y en tercer grado hasta el 10,000. Asimismo, para el caso de primer grado se aborda la expresión verbal y/o escrita de números ordinales del $1^{\circ}$ al $10^{\circ}$. Con respecto al uso de material manipulativo para abordar la enseñanza del valor posicional solo se hace uso de material de tipo no proporcional (Cid et al., 2003), lo anterior de acuerdo con Terigi y Wolman (2007) es una característica no tan conveniente porque promueve que el Sistema de Numeración Decimal se relacione con un sistema de numeración aditivo, y con ello se pierde la conceptualización del sistema.

Ahora, los procedimientos propuestos están ligados con el conteo de 1 en 1 , de 10 en 10, y de 100 en 100, la identificación de numerales, comparación de colecciones y la descomposición aditiva, los cuales emergen con la resolución 
de las tareas. Por otra parte, los conceptos abordados son cardinalidad, número ordinal, valor posicional, cifra, unidad, decena, centena, unidad de millar, y descomposición aditiva, los mismos no son trabajados en el texto de manera explícita. Con respecto a los argumentos estos se enfocan en el orden de la secuencia numérica y el valor posicional de números de hasta cuatro cifras. Finalmente, las proposiciones se relacionan implícitamente con las decenas, centenas, la descomposición aditiva, el orden de la secuencia numérica, la lectura-escritura de números naturales y ordinales.

\subsection{SigNIFICADOS DEL NÚMERO NATURAL}

Caracterizar los distintos significados del número natural en los libros de texto de matemáticas en Educación Primaria, puede contribuir al conocimiento del profesor sobre los contextos de uso promovidos en los distintos grados escolares. Lo cual podría servir como elemento de apoyo para el desarrollo del sentido numérico en los estudiantes (Cid et al., 2003; Godino et al., 2009; NCTM, 1989; SEP, 2011a) y la planificación docente, en el segundo periodo escolar.

Por ejemplo, para el significado de secuencia numérica, y particularmente sobre la introducción de los números naturales en los distintos grados escolares. En primer grado, se concentró la mayor cantidad de tareas (17 tareas), donde se aborda la expresión verbal, escrita y el orden de los números del 0 al 99, utilizando herramientas como la tabla-100, canciones infantiles, fichas, entre otras. En segundo grado, esta cantidad disminuye a cinco tareas, donde se contempla la expresión verbal, escrita y el orden de los números del 100 al 999. En tercer grado, ya no es presentada explícitamente, la secuencia numérica del 1000 al 10,000, pero si se utilizan números de cuatro cifras en la resolución de problemas.

Para el significado cardinal, y propiamente donde se incluyen tareas sobre el valor posicional de las cifras de un número. En primer grado, se trabaja de manera implícita el valor posicional de números de dos cifras utilizando fichas de colores. Mientras que, en segundo grado el valor posicional de los números de tres cifras y en tercer grado el valor posicional de números de hasta cuatro cifras. Lo anterior, sin utilizar o definir los términos de unidad, decena, centena y unidad de millar en ninguno de los grados escolares.

Por otra parte, el significado ordinal solo es abordado en primer grado, donde muestran la expresión verbal, escrita y el orden de los primeros 10 números ordinales. El significado simbólico es tratado en segundo y tercer grado con cinco tareas en total, donde se utilizan números de dos y tres cifras en tarjetas. 
Finalmente, para el significado operacional es abordado en primero, segundo y tercer grado, y los números se utilizan para realizar operaciones de suma y resta, así como comparación de números de dos y tres cifras, por ejemplo, en segundo grado se puede encontrar expresiones como 170+2>170.

El conocimiento de la existencia de los significados presentados en esta investigación, radica en la potencialidad que tiene en el uso que el docente de educación primaria pueda darle en el salón de clases. Dado que, si el profesor es consciente, por ejemplo, de la ausencia del tratamiento explícito de la expresión verbal y escrita de los números del 999 al 10, 000 puede proponer tareas para fortalecer este aspecto en la enseñanza-aprendizaje de este significado. Lo mismo sucede con el significado ordinal, dado que se puede proponer trabajar no solo con 10 números ordinales, sino que puede proponer incluir por lo menos los primeros veinte números ordinales. Además, entre más significados maneje el estudiante mejor será su compresión del número.

De manera general, se considera que mediante el análisis realizado se pudo caracterizar el significado pretendido en cada libro de texto. En ese sentido, el uso de los niveles de análisis de la actividad matemática para el análisis de tareas, permitió conocer en primer lugar los significados del número natural abordados en los libros de texto. Además, permitió mostrar un análisis pormenorizado de la actividad matemática que se pone en juego en la resolución de las tareas. Dicha información puede ser utilizada en la planeación docente, dado que permite tener una visión global del tratamiento del número natural en los tres primeros grados de la Educación Primaria.

\section{REFERENCIAS}

Aké, L. y Godino, J. D. (2018). Análisis de tareas de un libro de texto de primaria desde la perspectiva de los niveles de algebrización. Educación Matemática, 30(2), 171-201.

Alcalde, M., Pérez, I. y Lorenzo, G. (2014). Los números naturales en el aula de Primaria. Universitat Jaume I.

Alsina, A. y Vásquez, C. (2016). La probabilidad en educación primaria. De lo que debería enseñarse a lo que se enseña. UNO. Revista de Didáctica de las Matemáticas, 71, 46-52.

Becerra, A. y González, C. (2016). Los cuadriláteros en el libro oficial de Educación Primaria: del "Saber sabio" al "Saber para enseñar". Revista de Produçāo Discente em Educaçāo Matemática, 5(2), 18-29. 
Block, D. y Álvarez, A. M. (1999). Los números en primer grado: Cuatro generaciones de situaciones didácticas. Educación Matemática, 11(1), 57-76.

Braga, G. y Belver, J. L. (2016). El análisis de libros de texto: una estrategia metodológica en la formación de los profesionales de la educación. Revista Complutense de Educación, 27(1), 199-218.

Campistrous, L. A., Pastor, C., Pastor, G., Rizo, C. R. y Nava, A. (2013). El trabajo con números naturales en la escuela primaria mexicana. Universidad Autónoma de Guerrero.

Cárcamo, M., Díaz-Levicoy, D. y Ferrada, C. (2018). Los ejemplos en la enseñanza de las ecuaciones en libros de texto de Educación Primaria. REMAT: Revista Electrónica da Matemática, 4(1), 38-54. https://doi.org/10.35819/remat2018v4i1id2625 Ceballos, J. y Blanco, L. J. (2008). Análisis de los problemas de los libros de texto de Matemáticas para alumnos de 12 a 14 años de edad de España y de Chile en relación con los contenidos de proporcionalidad. Publicaciones, 38, 63-88.

Cid, E., Godino, J. D. y Batanero, C. (2003). Sistemas numéricos y su didáctica para maestros. Universidad de Granada.

Cortez, E. (2015). Análisis del libro de texto de 1er grado de primaria, reforma 1993: el caso de los números naturales (Tesis de licenciatura no publicada). Universidad Autónoma de Guerrero.

Díaz-Levicoy, D., Batanero, C., Arteaga, P. y Gea, M. M. (2015). Análisis de gráficos estadísticos en libros de texto de educación primaria española. UNIÓN. Revista Iberoamericana de Educación Matemática, 44, 90-112.

Díaz-Levicoy, D., Batanero, C., Arteaga, P. y López-Martín, M. D. (2015). Análisis de los gráficos estadísticos presentados en libros de texto de Educación Primaria chilena. Educaçāo Matematica Pesquisa, 17(4), 715-739.

Díaz-Levicoy, D., Giacomone, B. y Arteaga, P. (2017). Caracterización de los gráficos estadísticos en libros de texto argentinos del segundo ciclo de Educación Primaria. Profesorado. Revista de Currículum y formación del Profesorado, 21(2), 1-28.

Díaz-Levicoy, D., Morales-Garcia, L. y Rodríguez-Alveal, F. (2020). Las medidas de tendencia central en libros de texto de Educación Primaria en México. Revista Paradigma, 41, 706-721. https://doi.org/10.37618/PARADIGMA.1011-2251.2020.p706-729.id819

Giacomone, B., Díaz-Levicoy, D. y Godino, J. D. (2018). Análisis ontosemiótico de tareas que involucran gráficos estadísticos en Educación Primaria. Revista Digital Matemática, Educación e Internet, 18(1),1-13. https://doi.org/10.18845/rdmei.v18i1.3256

Godino, J. D. (2017). Construyendo un sistema modular e inclusivo de herramientas teóricas para la Educación Matemática. En J. M. Contreras, P. Arteaga, G. R. Cañadas, M.M. Gea, B. Giacomone y M. M. López-Martín (Eds.), Actas del Segundo Congreso International Virtual sobre el Enfoque Ontosemiótico del Conocimiento y la Instrucción Matemáticos (pp. 1-20). Universidad de Granada. 
Godino, J. D. y Batanero, C. (1994). Significado institucional y personal de los objetos matemáticos. Recherches en Didactique des Mathematiques, 14(3), 325-355.

Godino, J. D. y Ruiz, F. (2002). Geometría y su didáctica para maestros. Universidad de Granada. Godino, J. D., Beltrán-Pellicer, P., Burgos, M. y Giacomone, B. (2017). Significados pragmáticos y configuraciones ontosemióticas en el estudio de la proporcionalidad. En J. M. Contreras, P. Arteaga, G. R. Cañadas, M. M. Gea, B. Giacomone y M. M. López-Martín (Eds.), Actas del Segundo Congreso International Virtual sobre el Enfoque Ontosemiótico del Conocimiento y la Instrucción Matemáticos (pp. 1-13). Universidad de Granada.

Godino, J. D., Bencomo, D., Font, V. y Wilhelmi, M. R. (2006). Análisis y valoración de la idoneidad didáctica de procesos de estudio de las matemáticas. Paradigma, 22(2), 221-252.

Godino, J. D., Font, V., Konic, P. y Wilhelmi, M. R. (2009). El sentido numérico como articulación flexible de significados parciales de los números. En J. M. Peñas (Ed.), Investigación en el aula de Matemáticas. Sentido Numérico (pp. 117-184). SAEM Thales y Departamento de Didáctica de la Matemática de la Universidad de Granada.

Godino, J. D., Font, V., Wilhelmi, M. R. y Arrieche, M. (2009). ¿Alguien sabe qué es un número? UNIÓN. Revista Iberoamericana de Educación Matemática, 19, 34-46.

Godino, J. D., Font, V., Wilhelmi, M. R. y Lurduy, O. (2011). Why is the learning of the elementary arithmetic concepts difficult? Semiotic tools for understanding the nature of mathematical objects. Educational Studies in Mathematics, 77(2), 247-265.

Godino, J.D., Batanero, C. y Font, V. (2019). The onto-semiotic approach: implications for the prescriptive character of didactics. Fort the Learning of Mathematics, 39(1), 37-42.

Guillen, G., González, E. y García, M. G. (2009). Criterios específicos para analizar la geometría en libros de texto para la enseñanza primaria y secundaria obligatoria. Análisis desde los cuerpos de revolución. En M. J. González, M. T. González y J. Murillo (Eds.), Investigación en Educación Matemática XIII (247-258). SEIEM.

Kiener, F., Scaglia, S. y Gotte, M. (2013). Análisis del tratamiento del concepto de área en libros de texto de primaria. UNIÓN. Revista Iberoamericana de Educación Matemática, 36, 67-88.

Konic, P. M., Godino, J. D. y Rivas, M. A. (2010). Análisis de la introducción de los números decimales en un libro de texto, NÚMEROS. Revista de Didáctica de las Matemáticas, 74, 57-74.

Morales-Garcia, L. (2015). Identificación de ideas y/o procedimientos que niños de primer grado de primaria ponen en juego al resolver actividades que involucran la utilización del concepto de número natural (Tesis de licenciatura no publicada). Universidad Autónoma de Guerrero.

Navarro, C. (2015). Libros mexicanos de texto gratuito de matemáticas, reforma 2011: el caso de los números naturales y números fraccionarios (Tesis doctoral no publicada). Universidad Autónoma de Guerrero. 
NCTM (1989). Principles and Standards for school Mathematics. National Council of Teacher of Mathematics.

Orrantia, J., González, L. B. y Vicente, S. (2005). Análisis de los problemas aritméticos en los libros de texto de Educación Primaria. Infancia y Aprendizaje, 28(4), 429-451.

Rico, L., Marín, A., Lupiáñez, J. L. y Gómez, P. (2008). Planificación de las matemáticas escolares en secundaria. El caso de los Números Naturales. Suma, 58, 7-23.

Rodríguez-Nieto, C.A., Navarro, C., Castro Inostroza, A.N. y García-González, M.S. (2019). Estructuras semánticas de problemas aditivos de enunciado verbal en libros de texto mexicanos. Educación Matemática, 31(2), 75- 104.

Ruesga, P., Valls, F. y Rodríguez, T. (2006). Un instrumento para seleccionar libros de texto de matemáticas. Aplicación al bloque curricular de geometría. Revista Electrónica Interuniversitaria de Formación del Profesorado, 9(1), 1-13.

Salcedo, A. y Ramírez, T. (2016). Análisis de las actividades de probabilidad propuestas en textos escolares de primaria. Educaçāo Matematica Pesquisa, 18(1), 179-202.

Salgado, M. y Salinas, M.J. (2009). El número en los libros de texto de Educación Infantil. En M.J González, M.T. Gonzálezy J. Murillo (Eds.), Investigación en Educación Matemática XIII (pp.487-497). SEIEM.

SEP (2011a). Plan de estudios 2011. Secretaría de Educación Pública.

SEP (2011b). Programa de estudios. Primer grado. Secretaría de Educación Pública.

SEP (2011c). Programa de estudios. Segundo grado. Secretaría de Educación Pública.

SEP (2011d). Programa de estudios. Tercer grado. Secretaría de Educación Pública.

SEP (2014a). Desafíos Matemáticos. Primer grado. Libro para el alumno. Secretaría de Educación Pública.

SEP (2014b). Desafíos Matemáticos. Segundo grado. Libro para el alumno. Secretaría de Educación Pública.

SEP (2014c). Desafíos Matemáticos. Tercer grado. Libro para el alumno. Secretaría de Educación Pública.

Soto, S. (2016). Presentación de los números naturales en tres ediciones de libros de texto de primer grado de Primaria (Tesis de licenciatura no publicada). Universidad Autónoma de Guerrero.

Terigi, F. y Wolman, S. (2007). Sistemas de numeración: consideraciones acerca de su enseñanza. UNIÓN. Revista Iberoamericana de Educación Matemática, 43, 59-83.

\section{DANILO DÍAZ-LEVICOY}

Dirección: Departamento Matemática, Física y Estadística, Facultad de Ciencias Básicas, Universidad Católica del Maule, Avda. San Miguel 3605, Talca, Chile dddiaz01@hotmail.com

Teléfono: (+56) 978065371 